\title{
Classification of User Behaviour in Mobile Internet
}

\author{
Vaddempudi Srinidhi1)
}

\begin{abstract}
Smart devices such as android phones brings us the present mobile accessing of the internet and also making the mobile internet usage being increased rapidly from day by day and by the help of the mobile traffic data that was collected from the $2 \mathrm{G}$ and $3 \mathrm{G}$ networks in the metropolitan areas we will define the mobile user behaviour by the help of considering some of the aspects and mainly they are three aspects and the three aspects that we consider are such as Data Usage, Mobility Pattern and Application Usage, And then now we need to classify the mobile users into some of the different groups so as to study the resource consumption in the mobile internet. We will observe that the heavy traffic users and also the high mobility users will take in a huge data and also the huge amount of radio resources also simultaneously. Data usage and also the mobility patterns are very closely depends upon the application usage of the user as if it was mainly depend upon the user behaviour and the users can be combined by the help of the application usage behaviour and the categories of the application can be known by the way how to attract the users. Our analysis will provide the understanding the behaviour of the user in the mobile internet and also it will be helpful for the network designers to get or as to design the different kind of appropriate techniques in the resource provision and also the management of the mobility that which was based upon different types of applications.
\end{abstract}

Keywords : smart devices, android, mobile internet, 2G, 3G, network, behaviour.

\section{Introduction}

Smart devices such as android phones brings us the present mobile accessing of the internet and also making the mobile internet usage being increased rapidly from day by day and by the increase in the popularity of the behaviour of the user or the user behaviour has become an important one for the internet service providers that so as to perform the different tasks such as planning the capacity, management of the network, planning the network and also the network resources[1-3] In this paper we will try to know the behaviour of the users of the mobile by examining the three different important factors. They are:

Received(February 24, 2016), Review Result(1st: March 15, 2016, 2nd: April 15, 2016), Accepted(June 10, 2016)

${ }^{1}$ (Corresponding Author) Machine Intelligence Research Labs, India

email: srinidhisvsa@gmail.com 
1)Data Usage(mobile data resource)

2)Mobility Pattern(radio data resource)

3)Application usage(resource allocation)

Data usage was the fundamental one and it was normally related to the users, the mobile devices, different applications and also the type of the access of the networks and the type of access of the network can be either $2 \mathrm{G}$ or $3 \mathrm{G}$.

In the previous years it was given that the global internet had of about 18000 PB mobile data traffic and also considering the predictions of the author the mobile data traffic will be increased up to 61 percent from 2013 to 2018.

Application usage was the other important factor in the user behaviour for knowing the consumption of the network resource[4-5]. Unfortunately there was no method that was found for these type of analysis. The data that which was figured out by flow will contain the following data such as the user identifier that which is nothing but the phone number of the user and the timestamp(the time at which the flow of data will start)of the beginning of the flow and also how many no of bytes and packets that are there totally in the flow, uniform resource identifier, the server address and the name of the host and in these practice user may also shift from the cellular data service to the wi-fi so as to access the internet[6-8].

In such case the user will not have any phone number then we will get the unique identifier of the user by the help of packets and in these case we will analysis the behaviour of the user by the help of the cellular data usage.The users will be classified among the different groups as if according to their usage of volume of the traffic, mobility patterns and also the different types of the applications and in these we will define the two different types of groups such as

1) heavy traffic

2) normal traffic

So by the amount of traffic that was generated by the user behaviour we will be able to evaluate and also into four different types of groups such as

1)high mobility

2)normal mobility

3)low mobility 
4)non mobility

By these all groups we will try to understand or we will try to get the user behaviour.

\section{Proposed System}

\subsection{Traffic Data Usage Pattern}

Traffic usage patterns have been considered for two different types of users such as

1) heavy traffic users

2) normal users

And by the help these we get the result of the different usage patterns(data usage, mobility usage, application usage) of the users of the mobile. And the results of research was been saying that the less number of heavy traffic users will be contributed the large amount of traffic in the cellular network and also by these we can also say that the normal users will generate less amount of traffic in the cellular network and these was about the traffic data usage pattern and how we will analyze the traffic according to the user behaviour.

\subsection{Mobility Pattern}

The author has examined about the different mobility patterns in the mobile cellular networks and also the author has sates that he found that the dwell time distributions and also the inter arrival time distributions can be calculate by the help of the power law and the result will be mostly appropriately and it doesn't matter whether it is a day time or it is a night time or it might be in either rural or also in the urban areas.

And also examined the mobility pattern by different perspectives that which include:

1) the break down of the applications

2) the role of the users

3) the different types of devices

4) the characteristics of the diurnal 
The characteristics that were used are:

1) traffic load

2) mobility

3) resource efficiency

And these was about the mobility patterns and how it was analyzed.

\subsection{Browsing Behaviour Pattern}

The author found the user behaviour of different behaviour patterns can be classified into two types such as

1) Homogeneous

2) Heterogeneous

And also the author provided the information about the mobile user behaviour and as we already know that the user behaviour can be defined as:

1) data usage

2) mobility pattern

3) application usage

\section{Discussion}

\subsection{Analysis dimension}

We need to analyze the traffic of the data or the data traffic in different perspectives, such as:

1) Flow metrics

2) Time behaviour

3) User behaviour 
And the flow metrics consists of bytes, duration, number of the packet, flow number and also the user number and the graph is the representation of data and time interval in a week, days and also at the end of the week. And for doing the user behaviour analysis we consider the usage of data and the web service and also the mobility pattern.

\subsection{Data collection}

There are three different types of components in the mobile network, such as:

1)Mobile device

2)Access network

3)Core network

Mobile device is the one that which was an terminal that was connected to the mobile network and the $2 \mathrm{G}$ network accessing will be done by the BTS to BSC, where as 3G the data that was requested will be collected by the $B$ and then the result will be sent to radio network controller.

- Core network consists of SGSN and GGSN.

- Gn was in between the SGSN and GGSN.

- GGSN will send the information/data to the internet through Gi interface.

\subsection{Traffic characteristics overview}

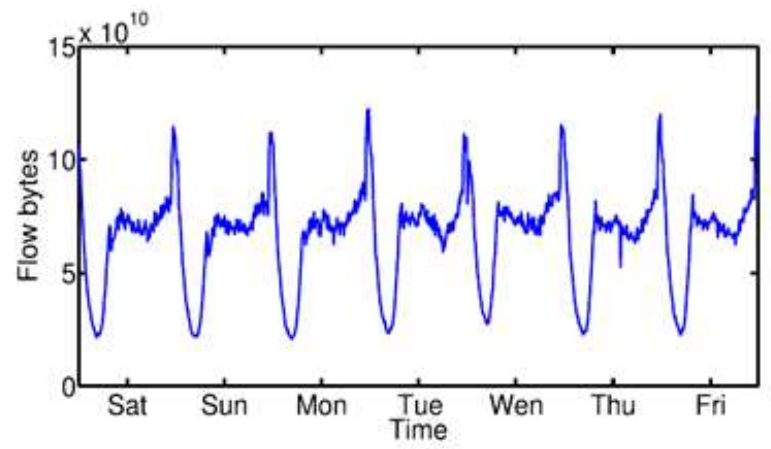

[Fig. 1] flow of bytes over a period of time. 


\subsection{Data Usage}

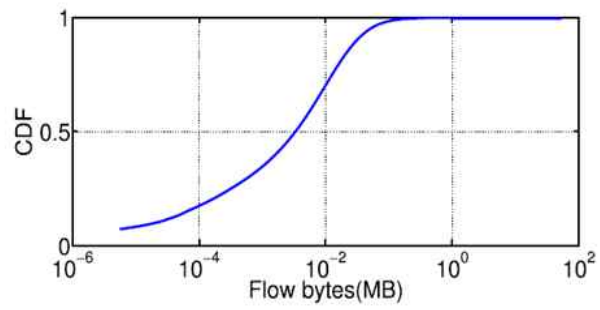

[Fig. 2] CDF of bytes usage of each in the week

In the graph shown above the curve that was shown was highly uneven and also the mobile users that were at top $1 \%$ of the user traffic can be defined as the heavy users and among the total the number of heavy users are very high in number.

So according to the graph that was drawn we can say that the heavy users are more in the percentage in the total amount of mobile data traffic.

\subsection{Application usage}

\begin{tabular}{|c|c|} 
Category & User Interest \\
\hline E-commerce & Online shopping, online payment \\
\hline Reading & Online reading, EBook \\
\hline Video & Online video \\
\hline Music & Online music \\
\hline Online gaming & Cyber games, network game \\
\hline Social network & Social network, sharing site, blog, Micro-blog \\
\hline News & Current news \\
\hline Mail & E-mail \\
\hline App store & Downloading the App or Android Applications \\
\hline Search & Search engine, Google \\
\hline Advertising & Advertisement \\
\hline
\end{tabular}

[Fig. 3] Application usage

These are the different application categories that we have consider for drawing the data set and we see one by one:

- In E-commerce user interest will be on online shopping and online payment.

- In Reading the user interest will be on online reading and Ebook. 
- In video the user interest will be on online video.

- In music the user interest will be on online music.

- In online gaming the user interest will be mainly on cyber games and network games.

- In social network the user interest will be on social network, blogs etc

- In news the user interest will be on current news. In mail the user interest in mainly in email.

\subsection{Mobility vs Data Usage}

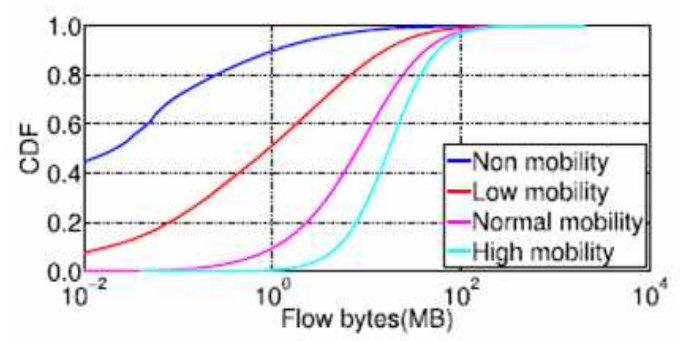

[Fig 4] CDF of generated traffic for each mobility group

The different kinds of the mobility groups as if they are of 4 different kinds such as:

1) non mobility users

2) low mobility users

3) normal mobility users

4) high mobility users

It is intriguing to discover how visit users switch between diverse cells, and whether there is any distinction in the middle of substantial and typical users as far as their mobility. We first draw a CDF of activity created by users in the week for every mobility bunch. We will consider the higher users mobility is, the more activity they create. This implies individuals tend to utilize their cell phone when they travel or on drive. All the more particularly, $90 \%$ of non mobility users produce under $1 \mathrm{MB}$ movement in the week. $51.30 \%, 9.44 \%$ and $0.55 \%$ users in every gathering of low, typical and high versatility produce under $1 \mathrm{MB}$ activity individually. 


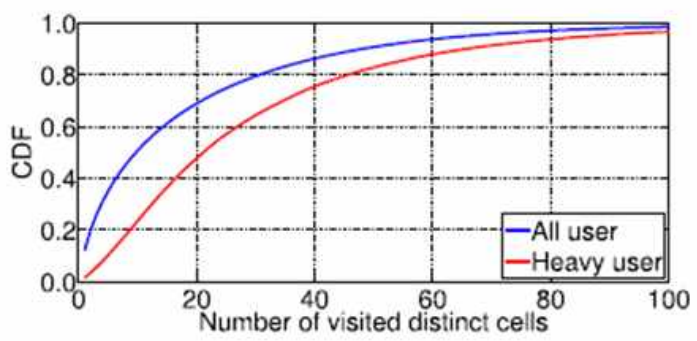

[Fig. 5] CDF of the number of visited distinct cells for all users and heavy users.

In normal, one non mobility user creates $1.30 \mathrm{MBtraffic}$ and one high portability client produces 28.11 MB activity. Furthermore, we further study the mobility of substantial client. The CDF of particular cells all clients and substantial users went to in the week. $1.45 \%$ of the substantial users are non mobility users, and there are $22.04 \%$ low mobility users, $59.42 \%$ typical versatility clients and $17.09 \%$ high mobility users. On account of all users, the comparing extents are $11.97 \%, 38.77 \%, 40.06 \%$ and $9.58 \%$ individually. We can reason that mobility users tend to visit more particular cells. As it were, overwhelming users as a rule additionally expend more radio asset notwithstanding the actuality they produce more movement than ordinary users.

\subsection{Application Usage vs Mobility}

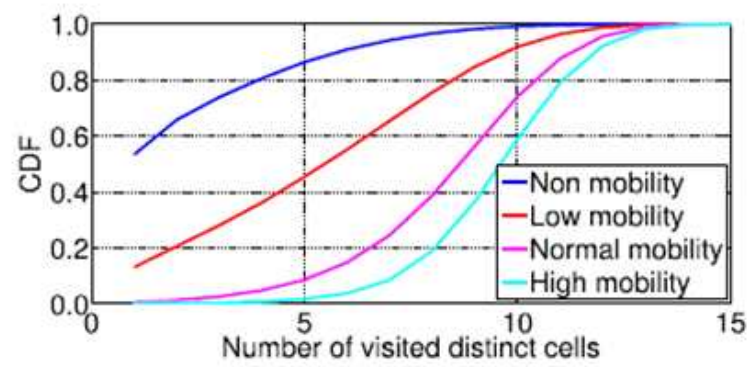

[Fig 6] CDF of the number of visited distinct cells

In this segment, we think about the relationship between the user favoured application classification and their mobility. In the first place, the relationship between the quantity of went to application classifications and user mobility design in the week is concentrated on, which shows the CDF of went by application classifications from distinctive mobility bunches in the week. As should be obvious, as a rule, the more the quantity of particular cells a user 
visit, the more assorted applications user went to in the week.

\subsection{Data usage vs application usage}

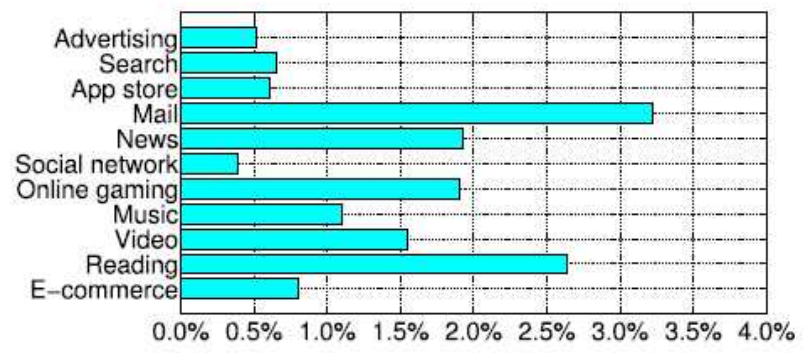

[Fig. 7] Data usage

The creators have displayed a few critical perceptions with respect to the relationship between application use and stream measurements, information utilization and mobility. Presently we consider to relate Data Usage with Web Usage to discover the inclinations of use classification for clients with diverse information use design.

They concentrate on substantial users as they are the ones that create the larger part of activity. After a nearby inspect of information utilization example of overwhelming users, we find that substantial users have a tendency to expend more information on application store, web gaming and music through cellular telephone. Informal community just takes $17.65 \%$ of aggregate substantial user activity, then again, on account of ordinary user movement, the rate is $55.65 \%$. Albeit overwhelming users just possess $1 \%$ of aggregate users, $3.22 \%$ mail clients, $2.64 \%$ perusing users and $3.22 \%$ news users were substantial users, and they contributed $21.91 \%$ mail activity, $13.65 \%$ perusing movement and $13.63 \%$ news movement separately.

That implies typical users don't check the mail, read books or news on the PDA as much as the overwhelming users.It better comprehend the effect of user conduct on system assets, we further study the relations between the three components (Data Usage, Mobility Pattern and Application Usage) in this area. Our study will be performed inside and between userbunches, e.g. the gatherings with diverse information utilization example and the gatherings with distinctive mobility design. Heavy users create most of the movement in Mobile Internet, prompting a noteworthy utilization of the system transfer speed. High mobility users cause high recurrence of hand off exercises, which, thusly, expend much additional radio assets. We call these two user bunches "Big Consumer of Resource". A profound comprehension of conduct of the huge purchasers could offer us some assistance with planning system 
improvement and asset distribution better.

\section{Conclusion}

In this paper, by utilizing the genuine traffic information gathered from portable Internet in an expansive metropolitan range of China, we concentrated on the portable client conduct with nitty gritty multi-measurement examination by concentrating on three elements - information utilization, mobility example and application use.

\section{References}

[1] S. Burford and S. Park, The impact of mobile tablet devices on human information behaviour, Journal of Documentation, (2014), Vol.70, No.4 pp.622-639.

[2] W. J. Potter, The state of media literacy, Journal of Broadcasting \& Electronic Media, (2010), Vol.54, No.4, pp.675-696.

[3] T. D. Wilson, Revisiting user studies and information needs, Journal of Documentation, (2006), Vol.62, No.6, pp.680-684.

[4] J. Hartley, K. McWilliam, J. Burgess, and J. Banks, The uses of multimedia: three digital literacy, Media International Australia, (2008), Vol.128, pp.59-72.

[5] P. S. Wei and H. P. Lu, Why do people play mobile social games? An examination of network externalities and of uses and gratifications, Internet Research, (2014), Vol.24, No.3, pp.313-331.

[6] S. M. Arteaga, V. M. Gonzalezb, S. Kurniawana, and R. A. Benavidesc, Mobile games and design requirements to increase teenagers' physical activity, Pervasive and Mobile Computing, (2012), Vol.8, No.6, pp.900-908.

[7] R. K. Baker and K. M. White, Predicting adolescents' use of social networking sites from an extended theory of planned behaviour perspective, Computers in Human Behavior, (2010), Vol.26, No.6, pp.1591-1597.

[8] I. Bose and X. Yang, Enter the Dragon: Khillwar's foray into the mobile gaming market of China, Communications of the Association for Information Systems, (2011), Vol.29, pp.551-564. 